\title{
Störungen der Zahnentwicklung bei Rattenembryonen nach Gaben von 6-Aminonicotinamid
}

Die Behandlung schwangerer Ratten mit 6-Aminonicotinamid (6-AN) führt in Abhängigkeit von Zeitpunkt und Dosis zu Resorptionen, Retardierungen und Missbildungen ${ }^{1}$. Vorwiegend Gaumenspalten sind als Folge dieser Behandlung beschrieben worden ${ }^{2,3}$. Im biochemischen Bereich führt 6-AN wahrscheinlich zu einer Störung des Glukosestoffwechsels über eine kompetetive Hemmung NADP-abhängiger Reaktionen im Pentosephosphatweg, wobei allerdings noch andere Enzymsysteme sekundär betroffen sein können $\mathbf{4}^{4}$. Über Missbildungen an Zähnen ist in diesem Zusammenhang noch nicht berichtet worden.

Schwangere Ratten (Stamm FW 68) erhielten vom Tag 13 bis Tag 17 je 1 i.p.- Injektion mit $8 \mathrm{mg} / \mathrm{kg}$ 6-AN. Am Tag 0 wurden die Tiere bei umgekehrtem TagNachtrhythmus von 7.30 bis 10.00 h verpaart. Am Tag 18 erfolgte die Fixierung in gepuffertem Formol für die lichtmikroskopische oder in Karnovskyscher Lösung für die elektronenmikroskopische Untersuchung.

Bei der lichtmikroskopischen Betrachtung fand sich eine deutliche Hemmung der Zahnentwicklung. Am Tag 18 ist bei den Nagezähnen in den Kontrollschnitten die Zahnglocke bereits typisch ausgebildet, der epitheliale Anteil hat sich in äusseres und inneres Schmelzepithel sowie in Schmelzpulpa differenziert und die Odontoblasten beginnen sich in det Zahnpapille zu ordnen. Eine organische Matrix ist noch nicht vorhanden. Nach Gaben von 6-AN wird die Zahnentwicklung auf der Reifungsstufe des Injektionszeitpunktes arretiert: Nach Injektion am Tag 13 findet sich auch am Tag 18 nur der Beginn der Zahnleistenbildung. Injektionen an den folgenden Tagen resultieren in einer Blockierung auf einem Stadium der
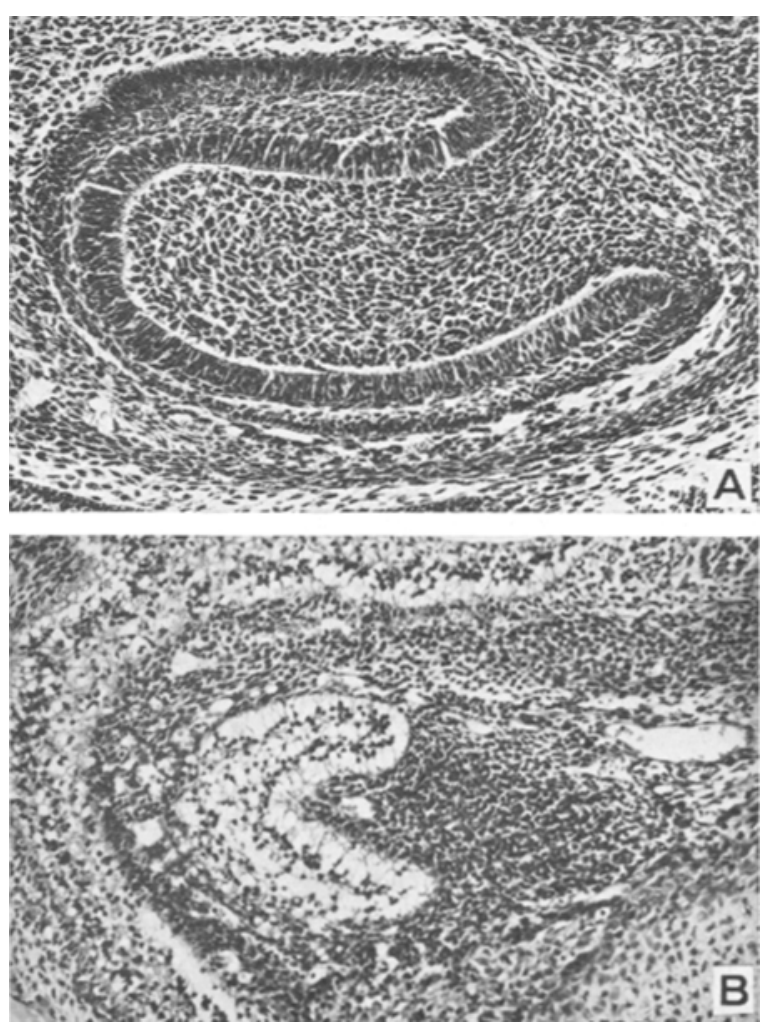

Fig. 1. a) Unterer Incisivus eines Rattenembryos vom Tag 18. b) Unterer Incisivus eines Rattenembryos vom Tag $18 \mathrm{mit} 8 \mathrm{mg} / \mathrm{kg}$ 6-AN gespritzt am Tag $15+7$ Std. $\times 350$. frühen Glockenbildung, die nicht dem Tag 18 entspricht. Die Injektion am späten Tag 16 und am Tag 17 lässt zwar eine typische Zahnglocke erkennen, jedoch findet sich keine deutliche Gliederung des Epithels.

Das Epithel ist unter dem Lichtmikroskop vakuolig verändert. Elektronenmikroskopische Untersuchungen zeigen, dass für dieses lichtmikroskopische Bild eigentümliche und lokalisierte Schwellung der endoplasmatischen Hohlräume verantwortlich sind. Besonders betrof-

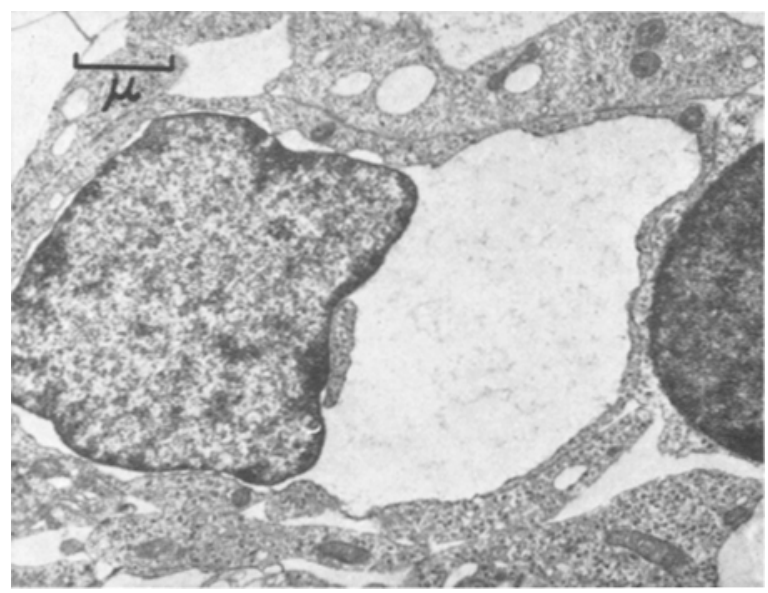

Fig. 2. Elektronenmikroskopisches Bild einer Epithelzelle aus der Anlage des unteren Incisivus am Tag 18 nach Injektion von $8 \mathrm{mg} / \mathrm{kg}$ 6-AN am Tag $15+7$ Std. Extreme Erweiterung der perintucleären Cisterne. $\times 10000$.

fen ist die perinukleäre Cisterne. Sie erweitert sich häufig so stark, dass das restliche Cytoplasma mit den unveränderten Zellorganellen an die Peripherie gedrängt wird und der Kern nur an Verbindungsfäden im Zentrum dieser Blase aufgehängt ist.

Diese Befunde weisen auf eine Schädigung des epithelialen Anteils der Zahnanlage durch 6-AN hin, die zu einer Blockierung des weiteren Wachstums führt.

Summary. Application of 6-aminonicotinamide to rats during pregnancy produced malformations, resorption and retardation. A dose of $8 \mathrm{mg} / \mathrm{kg} \mathrm{6-AN}$ between day 12 and 17 of pregnancy can affect the development of teeth germ. Electron microscopic studies reveal disturbances of the epithelial part of the teeth germ only, with an extreme balooning of the perinuclear cistern.

\section{H. J. Merkér und L. Nováx}

\section{Anatomisches Institut}

der Freien Universität Berlin, Königin-Luise-Strasse 15, D-1-Berlin 33 (Deutschland), und Stomatologische Klinik der Medizinischen Fakultät dev Karls-Universität, Hradec Králové (CSSR), 23. März 1970.

I H. Turbow und J. G. Chamberlain, Teratology 1, 103 (1968).

a J. G. Chamberlain und M. Nelson, J. exp. Zool. 153, 285 (1963). 3 J. G. Chamberiain, Anat. Rec. 156,31 (1966).

4 H. Herken, K. Lange und H. Kolbe, Biochem, biophys. Res. Commun. 36, 93 (1969).

5 E. Köhler und D. Neubert, Naunyn-Schmiedebergs Arch. exp. Path. Pharmak. 260, 154 (1968). 